\title{
Safety and Efficacy of Flumazenil for Reversal of Iatrogenic Benzodiazepine-Associated Delirium Toxicity During Treatment of Alcohol Withdrawal, a Retrospective Review at One Center
}

\author{
Philip W. Moore - J Ward Donovan • Keith K. Burkhart • \\ Jeffrey A. Waskin • Michelle A. Hieger • Audrey R. Adkins • \\ Yijin Wert • David A. Haggerty • J. J. Rasimas
}

Published online: 12 March 2014

(C) American College of Medical Toxicology 2014

\begin{abstract}
Both alcohol withdrawal syndrome (AWS) and benzodiazepines can cause delirium. Benzodiazepineassociated delirium can complicate AWS and prolong hospitalization. Benzodiazepine delirium can be diagnosed with flumazenil, a GABA-A receptor antagonist. By reversing the effects of benzodiazepines, flumazenil is theorized to exacerbate symptoms of AWS and precludes its use. For patients being treated for alcohol withdrawal, flumazenil can diagnose and treat benzodiazepine delirium without precipitating serious or life-threatening adverse events. Hospital admission records were retrospectively reviewed for patients with the diagnosis of AWS who received both benzodiazepines and
\end{abstract}

P. W. Moore $(\bowtie) \cdot J$. W. Donovan · K. K. Burkhart · Y. Wert •

D. A. Haggerty $\cdot$ J. J. Rasimas

Department of Internal Medicine, Harrisburg Hospital,

PinnacleHealth, Harrisburg, PA 17101, USA

e-mail: pwmoore@pinnaclehealth.org

J. W. Donovan · K. K. Burkhart • J. J. Rasimas

Department of Emergency Medicine, Pennsylvania State University

College of Medicine, Hershey, PA, USA

K. K. Burkhart

Office of Translational Sciences, Office of Clinical Pharmacology,

Food and Drug Administration, Center for Drug Evaluation and

Research, Silver Spring, MD, USA

J. A. Waskin · M. A. Hieger · A. R. Adkins

Department of Emergency Medicine, Memorial Hospital,

York, PA, USA

D. A. Haggerty

Department of Emergency Medicine, Good Samaritan Hospital,

Lebanon, PA, USA

J. J. Rasimas

National Institutes of Health, NIMH, Bethesda, MD, USA flumazenil from December 2006 to June 2012 at a universityaffiliated inpatient toxicology center. The day of last alcohol consumption was estimated from available blood alcohol content or subjective history. Corresponding benzodiazepine, flumazenil, and adjunctive sedative pharmacy records were reviewed, as were demographic, clinical course, and outcome data. Eighty-five patients were identified (average age 50.3 years). Alcohol concentrations were detectable for 42 patients with average $261 \mathrm{mg} / \mathrm{dL}(10-530 \mathrm{mg} / \mathrm{dL})$. Eighty patients were treated with adjunctive agents for alcohol withdrawal including antipsychotics $(n=57)$, opioids $(n=27)$, clonidine $(n=35)$, and phenobarbital $(n=23)$. Average time of flumazenil administration was 4.7 days (1-11 days) after abstinence, and average dose was $0.5 \mathrm{mg}(0.2-1 \mathrm{mg})$. At the time of flumazenil administration, delirium was described as hypoactive $(n=21)$, hyperactive $(n=15)$, mixed $(n=41)$, or not specified $(n=8)$. Response was not documented in 11 cases. Sixty-two (72.9 \%) patients had significant objective improvement after receiving flumazenil. Fifty-six patients required more than one dose (average 5.6 doses). There were no major adverse events and minor adverse effects included transiently increased anxiety in two patients: 1 patient who received $0.5 \mathrm{mg}$ on abstinence day 2 and another patient who received $0.2 \mathrm{mg}$ flumazenil on abstinence day 11. This is the largest series diagnosing benzodiazepine delirium after AWS in patients receiving flumazenil. During the treatment of AWS, if delirium is present on day 5, a test dose of flumazenil may be considered to establish benzodiazepine delirium. With the limited data set often accompanying patients with AWS, flumazenil diagnosed benzodiazepine delirium during the treatment of AWS and improved impairments in cognition and behavior without serious or life-threatening adverse events in our patients. 
Keywords Alcohol withdrawal syndrome/delirium tremens . Benzodiazepine delirium/benzodiazepine toxicity . Hypoactive delirium/hyperactive delirium/agitated delirium . Flumazenil diagnostic/flumazenil therapeutic . Benzodiazepine/lorazepam/diazepam/midazolam . Pharmacovigilence

\section{Introduction}

Benzodiazepines are $\gamma$-aminobutyric acid type A (GABA-A) receptor agonists which bind to sites in the central nervous system (CNS) and exert sedative and amnestic effects [1-3]. These inhibitory CNS effects are useful during the treatment of alcohol withdrawal syndrome (AWS), but can also result in dose-dependent CNS depression and even frank delirium. Flumazenil, a benzodiazepine receptor antagonist, effectively reverses benzodiazepine-induced CNS depression via competitive inhibition at the GABA-A receptor-binding site [2-8]. Flumazenil has been beneficial for expediting patient recovery from anesthesia [9-11] and was previously studied in 519 patients at our center; of which, 15 were diagnosed with alcohol withdrawal [12].

Complication rates after flumazenil administration have been reported anywhere from 0.1 to $23.4 \%$ [13-16]. Adverse events such as anxiety, agitation, seizures, and arrhythmias have been described [12-14, 17-19]. While most of the existing literature points toward the safety of flumazenil use in cases of acute benzodiazepine overdose and postoperative sedation, there has been only one published study using flumazenil for the treatment of a complication of AWS, benzodiazepine delirium. However, one earlier retrospective study of over 500 patients who received flumazenil during AWS treatment revealed no seizures or arrhythmias [18].

This retrospective study has, as its foundation, a bedside toxicology practice which, on the basis of history and physical examination, employs flumazenil frequently in patients with altered mental status suspected to be caused by sedatives. A brief description of this practice applied to patients with AWS is necessary to frame the communicated results. At this center, we use flumazenil to diagnose and treat benzodiazepine delirium during AWS after sedatives have been employed earlier in the hospital course. If (a) the patient's vital signs have normalized and stabilized, (b) neurologic exam reveals normalto-diminished deep tendon reflexes, and (c) wakefulness and attention are impaired, all hours after the peak effect of the last dose of benzodiazepine (typically IV lorazepam), then benzodiazepine delirium is suspected. Flumazenil is administered to such patients over 30 second into a running IV line (in order to avoid confusing a response to pain from drug administration with a true CNS effect). The first dose is 0.2 to $0.3 \mathrm{mg}$ if there are concerns about the clinical consequences of rapid awakening, but $0.5 \mathrm{mg}$ is the typical initial dose. If a patient's mental status improves after the initial dose, benzodiazepines are discontinued and an order on the hospital electronic medical record system is placed to administer $0.5 \mathrm{mg}$ flumazenil using prn dosing schedules up to every $1 \mathrm{~h}$ as needed for delirium or a Riker score $<4$ or $>4$. If the patient is receiving doses every hour, the effect fails to last 1 hour, or there is a less than 1:4 nurse-to-patient ratio, we consider increasing the dose of flumazenil to $1 \mathrm{mg}$ to increase the duration of effect, although prior studies suggest this prolonged effect occurs with doses larger than $3 \mathrm{mg}$ [20]. If there is no response to flumazenil, we pursue other causes for delirium. Our objective was to describe our clinical experience with flumazenil for the treatment of benzodiazepine delirium during the treatment of AWS with a larger cohort.

\section{Methods}

We obtained institutional review board approval to describe our experience and results with this clinical practice protocol. Approval was given for a retrospective chart review of all patients seen by the toxicology service to examine outcomes of administration of flumazenil to patients treated with benzodiazepines for alcohol withdrawal. We identified cases by querying the electronic pharmacy database for patients who received both benzodiazepines (diazepam, lorazepam, and/or midazolam) and flumazenil during their admission. From this list, patients with alcohol withdrawal were identified by reviewing attending progress notes and discharge diagnoses. Exclusion criteria were defined as patients without a diagnosis of alcohol withdrawal, patients who did not receive flumazenil, and patients who did not receive benzodiazepines before receiving flumazenil.

Physician notes were the preferred source of data to collect the following demographic information: age, gender, prescribed medications, alcohol use history, day of last drink, serum alcohol concentration at admission, hospital admission date and time, and initial benzodiazepine dose, date, and time as well as other adjunctive medications (i.e., sedatives, opioids, and antipsychotics) received before the first dose of flumazenil. Documentation from the physician care team was also used to confirm diagnoses of delirium and assign phenomenological subtypes (i.e., hypoactive, hyperactive, or mixed) [21-23] based on the Riker scale $[24,25]$ or patient description if the Riker scale was not found. Delirium was diagnosed by the DSM-IV-TR criteria. The Riker sedation-agitation scale was used to categorize delirium $[25,26]$ as hypoactive if the Riker score $<4$, hyperactive if $>4$, and mixed delirium if Riker score fluctuating between $<4$ and $>4$. The qualifier NOS was used for patients whose records did not contain a Riker score at the time of the first flumazenil administration. 
Comorbidities such as history of seizures were noted. Seizure history was further delineated as active (seizure documented by EMS, ED, or occurred during hospitalization) or inactive (seizure disorder based on medical history and use of antiepileptic medication). Pharmacy electronic medical records were used to record flumazenil and benzodiazepine first and last dose date and time and number of total flumazenil and benzodiazepine doses and amounts given (this system is electronic which requires a patient's wristband be scanned before administration, increasing accuracy of both identity and timing of medication administration).

Immediately following the first dose of flumazenil, attending physician notes were reviewed for a change in mental status within 1 to 5 minutes. A positive response was considered in those with improved mental status from agitated to calm or from sedate to awake (i.e., hypoactive delirium attributed to benzodiazepines). Positive-response data were corroborated by subsequent orders to discontinue benzodiazepines following the test dose of antidote. Resident, fellow, and nursing notes served as supplements to attending documented response data. Pharmacy records showing discontinuation of benzodiazepines and reordering flumazenil as needed for delirium symptoms and/or to enhance participation in care further corroborated positive-response data. If no response to the first dose of flumazenil was found in the initial note and the patient received additional flumazenil doses, details documented from the subsequent doses regarding mental status changes were used for response data. A negative response was considered to be increased anxiety, agitation, tachycardia, hypertension, arrhythmias, or seizures. Patients who were unchanged after flumazenil administration were tabulated as such on the basis of clinical documentation as well. Continuation of benzodiazepines after a first and only dose of flumazenil, as recorded in pharmacy records, was deemed supporting evidence for a negative response, unchanged response, or unknown effect. If no documented response to flumazenil could be found, patients were categorized as having an unknown response to flumazenil. Patients with missing data were included, but the specific missing information was noted. A response was expected within minutes of flumazenil administration; if any positive symptom change occurred more than $5 \mathrm{~min}$ from the first dose of flumazenil, the effect was considered to be unrelated to flumazenil administration.

Four reviewers were trained and performed the chart reviews after collectively viewing sample charts. The principal investigator verified accuracy of $75 \%$ of the charts reviewed; if the error rate exceeded $5 \%$, the investigator was provided further education regarding the data collection process and the collected data were subjected to an accuracy audit before being included in analyses.

Microsoft Excel ${ }^{\circledR}$ (2011) was used to generate figures comparing flumazenil dose to effect as well as abstinence day at the time of the first dose of flumazenil. Data was statistically analyzed using SAS version 9.2. Proportions were computed for categorical variables. Mean, median, and range were calculated for continuous variables. The descriptive statistics included flumazenil dose to effect as well as abstinence day at the time of the first dose of flumazenil. Fisher's exact test was applied to compare the frequency of patients experiencing improvement after receiving flumazenil between the hyperactive group and the hypoactive and mixed delirium group. A $p$ value of less than 0.05 was considered significant.

\section{Results}

Two hundred fifty-six patients received both flumazenil and benzodiazepines, and 85 patients were included in the study. Average patient ages can be found in Table 1. Fourteen patients $(16 \%)$ were prescribed benzodiazepines for home use prior to coming to the hospital and seven patients $(8 \%)$ were on home tricyclic antidepressants. Fifteen patients $(18 \%)$ had seizures noted pre-hospital, while in the emergency department, or on abstinence day 1 . Nine patients (11\%) reported history of alcohol withdrawal seizure but did not have recurrence during hospitalization. Two patients $(2 \%)$ had history of seizure disorder without mention of alcohol. At the time of admission, serum alcohol was detectable in 42 patients (49\%) with a mean concentration of $261 \mathrm{mg} / \mathrm{dL}$ $(10-530 \mathrm{mg} / \mathrm{dL})$. Two of these intoxicated patients received benzodiazepines while in the emergency department for disinhibition and later, when evaluated by the toxicology service, received flumazenil to exclude contribution from benzodiazepines, and both had some improvement without adverse effects. Fifteen patients did not have initial alcohol levels available for review. The frequency of benzodiazepine administration during hospitalization can be found in Tables 2 and 3 . Eighty patients (94\%) were treated with adjunctive agents (Table 4). The average abstinence day (ethanol day free) of initial flumazenil administration was 4.7 days (1-11 days, Fig. 1). The median initial dose of flumazenil was $0.5 \mathrm{mg}$ $(0.2-0.5)$. Negative responses occurred in two patients $(2.3 \%)$, and both were described as increased anxiety or transiently increased agitation.

Mental status response in patients with AWS and possible benzodiazepine delirium after receiving $0.2 \mathrm{mg}(n=13)$ or $0.3 \mathrm{mg}(n=1)$ of flumazenil were improved for 10/14 (71\%)

Table 1 Ages of patients with alcohol withdrawal $(n=85)$

\begin{tabular}{lll}
\hline $\begin{array}{l}\text { Number of } \\
\text { patients }\end{array}$ & $\begin{array}{l}\text { Mean, median (range) } \\
\text { age (years) }\end{array}$ & $\begin{array}{l}\text { Number with age }>65 \\
\text { (range) }\end{array}$ \\
\hline Total $(n=85)$ & $50.3,50(27-81)$ & $4(67-81)$ \\
Men $(n=71)$ & $51,50(30-81)$ & $4(67-81)$ \\
Women $(n=14)$ & $47,46(27-64)$ & 0 \\
\hline
\end{tabular}


Table 2 Mean, median, and range of benzodiazepine doses received prior to first dose of flumazenil $(n=85)$

\begin{tabular}{lllll}
\hline Benzodiazepine & Number & Mean $(\mathrm{mg})$ & Median $(\mathrm{mg})$ & Range (mg) \\
\hline Lorazepam & 85 & 167 & 120 & $1-857$ \\
Diazepam & 60 & 180 & 145 & $5-596$ \\
Midazolam & 11 & 12 & 12 & $2-29$ \\
\hline
\end{tabular}

and unchanged for 3/14 (21\%). The negative effect in one patient was described as transiently increased agitation; this patient received flumazenil on abstinence day 11 . Mental status response in patients with AWS and possible benzodiazepine delirium after receiving $0.5 \mathrm{mg}$ of flumazenil $(n=71)$ were improved for $56(79 \%)$ and unchanged for 14 $(20 \%)$. The negative effect in one patient was described as transiently increased anxiety; this patient received flumazenil on abstinence day 2 .

At the time of flumazenil administration, delirium was described as hypoactive (over-sedate) $(n=21,25 \%)$, agitated $(n=15,18 \%)$, mixed $(n=41,48 \%)$, or NOS $(n=8,9 \%)$ and can be found in Table 5. Sixty-two patients (72.9\%) had objective improvement immediately after flumazenil administration. Riker scores were found pre- and post-flumazenil for seven of the patients with hypoactive delirium (pre/post flumazenil: $2 / 4,1 / 1,2 / 3,2 / 3,2 / 4,2 / 4,1 / 4)$. The remaining 14 patients had clearly documented response but not in the Riker format, e.g., patient awake and now able to use incentive spirometer or patient more awake after flumazenil. Patients with a purely hyperactive delirium were less likely to have a positive response to flumazenil than other delirium subtypes ( $p=0.01$ ), but over half of those patients showed neurobehavioral improvement. For the group with hyperactive delirium, two patients were found to have Riker scores recorded pre- and post-flumazenil administration. The remaining 13 patients had clearly documented response but not in the Riker format, e.g., patient more oriented, much less agitated and able to follow directions, agitation improved, or improved mental status.

Fifty-six patients were administered more than one dose of flumazenil. The mean total number of doses was 8 , and the mean total dose was $4.6 \mathrm{mg}$ over a mean of 2 days. Table 3
Table 4 Adjunctive agents received for hospitalized patients with alcohol withdrawal prior to first dose of flumazenil $(n=80)$, for patients who received more than one dose of flumazenil during hospitalization $(n=56)$, and for patients whom received only one dose of flumazenil $(n=24)$

\begin{tabular}{llll}
\hline Medication & $\begin{array}{l}\text { Number (\%) } \\
\text { patients receiving } \\
\text { adjunctive agents } \\
\text { before first dose } \\
\text { FMZ }(n=80)\end{array}$ & $\begin{array}{l}\text { Number }(\%) \\
\text { patients receiving } \\
\text { adjunctive agents } \\
\text { and who received } \\
>1 \text { FMZ }(n=56)\end{array}$ & $\begin{array}{l}\text { Number }(\%) \\
\text { patients receiving } \\
\text { adjunctive agents } \\
\text { and who received } \\
\text { only one dose of } \\
\text { FMZ }(n=24)\end{array}$ \\
\hline $\begin{array}{l}\text { Phenobarbital } \\
\text { Opioids }\end{array}$ & $23(27)$ & $20(36)$ & $3(13)$ \\
Clonidine & $35(41)$ & $20(36)$ & $6(25)$ \\
Haloperidol & $57(67)$ & $25(45)$ & $8(33)$ \\
\hline
\end{tabular}

provides more details regarding the subset of patients who showed benefit from more than one dose of flumazenil. None of the 85 patients in our study had a documented seizure or arrhythmia after administration of flumazenil, and none suffered serious agitation which required treatment. During the dates of this study, our pharmacy and therapeutics committee allowed flumazenil to be administered on any telemetry floor as long as the patient was monitored on telemetry and assessed with pre- and post-administration neurological and vital sign checks, and as per floor protocol.

Table 4 describes patients who received adjunctive agents (non-benzodiazepine medications) for treatment of alcohol withdrawal and who later received flumazenil $(n=80)$. Twenty-four of these patients only received one dose of flumazenil and 56 patients received more than one dose of flumazenil. The most common adjunctive agents were haloperidol, followed by clonidine and phenobarbital, respectively.

Sixteen of our patients were treated in the intensive care unit during hospitalization for respiratory failure requiring endotracheal intubation and mechanical ventilation. Four of these patients were comatose or had unresponsive mental status (one baclofen poisoning, one carisoprodol poisoning, and two post-ictal). Four patients had severe agitation related to alcohol withdrawal, phencyclidine, and/or cocaine intoxication. Three patients experienced hypoxic respiratory failure

Table 3 Mean, median, and range of benzodiazepine doses $(\mathrm{mg})$ received prior to first dose of flumazenil for patients who received more than one dose of flumazenil $(n=56)$ and for patients whom received only one dose of flumazenil $(n=29)$

\begin{tabular}{lllll}
\hline Benzodiazepine & $\begin{array}{l}\text { Number (\%) patients who } \\
\text { received }>1 \text { FMZ }(n=56)\end{array}$ & $\begin{array}{l}\text { Mean, median (range) } \\
\text { BZD received }\end{array}$ & $\begin{array}{l}\text { Number (\%) Patients who received } \\
\text { ones dose FMZ }(n=29)\end{array}$ & $\begin{array}{l}\text { Mean, median (range) } \\
\text { BZD received }\end{array}$ \\
\hline Lorazepam & $56(100)$ & $198,175(8-857)$ & $29(100 \%)$ & $108,44(1-521)$ \\
Diazepam & $46(82)$ & $190,160(10-596)$ & $11(38 \%)$ & $206,80(5-485)$ \\
Midazolam & $9(16)$ & $12,9(2-29)$ & $1(3 \%)$ & 14 \\
\hline
\end{tabular}

$B Z D$ benzodiazepine, FMZ flumazenil 
Fig. 1 Abstinence day and initial amount of flumazenil administered to patients with suspected benzodiazepine delirium $(n=85)$. There were no major adverse events, and minor adverse effects were transiently increased anxiety for one patient who received $0.5 \mathrm{mg}$ on abstinence day 2 and another patient who received $0.2 \mathrm{mg}$ on abstinence day 11

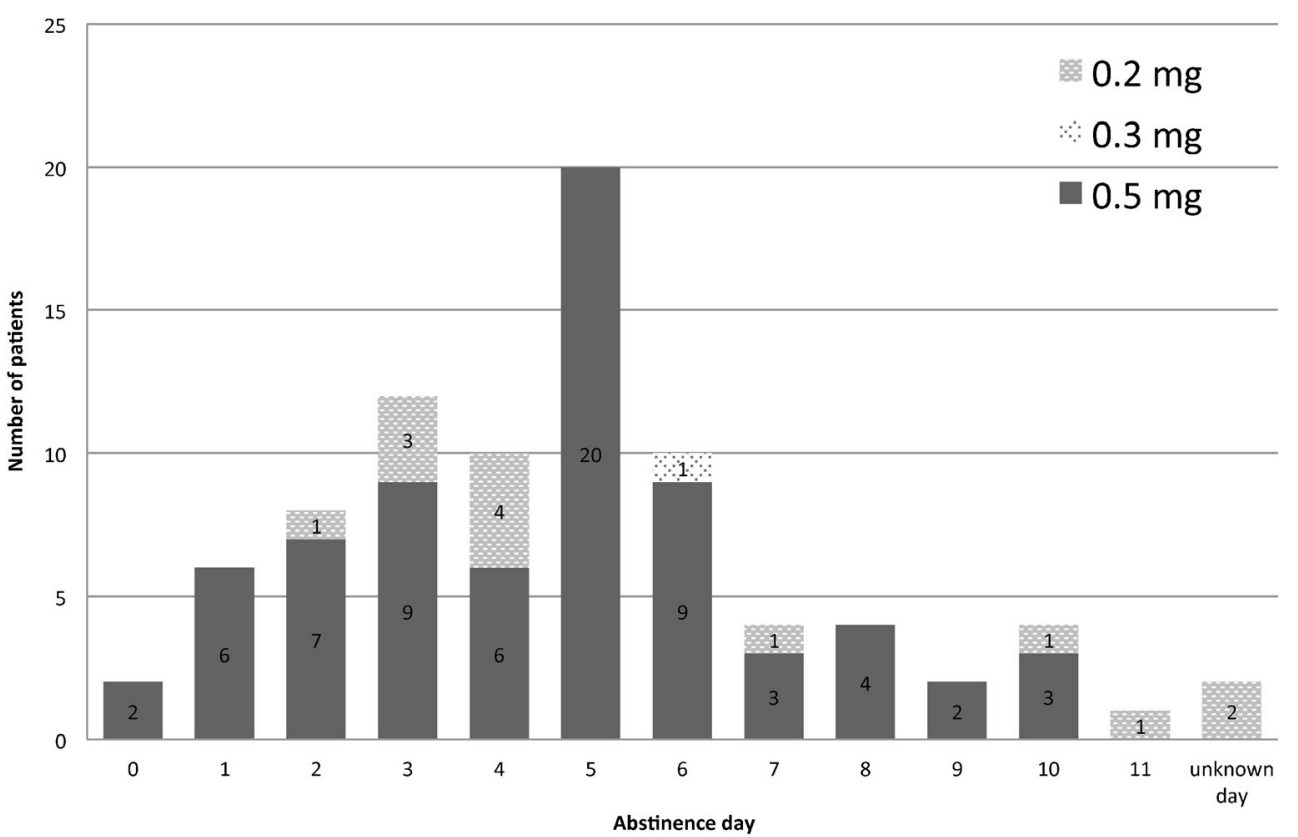

(one with pneumonia, one with pulmonary edema, one from sedation during course of alcohol withdrawal). Three patients had endotracheal intubation to facilitate other procedures (one for endoscopy, two for central lines). One patient had endotracheal intubation for airway edema.

One patient received test doses of flumazenil at different points of hospitalization. A 62 year old was admitted with a blood alcohol concentration of $320 \mathrm{mg} / \mathrm{dL}$, developed hypoxic respiratory failure, and was given a test dose of flumazenil on abstinence day 2. Because of pulmonary edema and aspiration pneumonitis, he was intubated for respiratory support and diuresis. He was extubated on abstinence day 9, and because of mixed delirium suspected secondary to lorazepam, he received flumazenil 10 hours later. He had a positive response with greater wakefulness, resolution of confusion, and restoration of his ability to participate in care including the use of incentive spirometry. Flumazenil was continued on an as-needed basis over the next 4 days in accordance with our standard practice; the patient received a total of 14 doses.

\section{Discussion}

This study was designed to investigate the risks and benefits of flumazenil use for hospitalized patients who experience adverse CNS effects from benzodiazepine medications during the treatment of AWS. Many of our patients had measured alcohol concentrations, which along with history assisted us to determine abstinence day. As both AWS and the treatment for it are recognized precipitants of delirium, we strove to elucidate how a benzodiazepine antagonist may be useful for differentiating between the two during hospital treatment.

We used flumazenil to diagnose benzodiazepine delirium in patients with hypoactive, hyperactive, and mixed delirium; a plurality of our patients had mixed delirium. In our bedside practice, flumazenil has a favorable risk-benefit ratio regardless of the subtype, but patients with pure hyperactive delirium were less likely to have a positive response after flumazenil when compared against a combined group containing hypoactive and mixed delirium subtypes $(p=0.0113)$. This finding is consistent with what clinical pharmacologic

Table 5 Delirium type and mental status response after flumazenil

\begin{tabular}{|c|c|c|c|c|c|}
\hline Delirium type & Number & Positive (\%) & Unchanged (\%) & Unknown (\%) & Negative \\
\hline Hypoactive & $21(25 \%)$ & $18(85.7)$ & $2(9.5)$ & $1(4.8)$ & 0 \\
\hline Hyperactive & $15(18 \%)$ & $8 *(53.3)$ & $4(26.7)$ & $2(13.3)$ & $1(6.7 \%)$ \\
\hline Mixed & $41(48 \%)$ & $35(85.4)$ & $4(9.8)$ & $1(2.4)$ & $1(2.4 \%)$ \\
\hline \multirow[t]{2}{*}{ NOS } & $8(9 \%)$ & $1(12.5)$ & $2(25)$ & $5(62.5)$ & 0 \\
\hline & 85 & $62(72.9)$ & $12(14.1)$ & $9(10.5)$ & $2(2.3 \%)$ \\
\hline
\end{tabular}

${ }^{*} p=0.0113$ related to the decreased likelihood of positive response in hyperactive delirium compared to the combination of hypoactive and mixed delirium subtypes 
experience would suggest, as patients suffering the effects of excess GABA agonism are typically less energetic or consistently agitated. Some individuals do, however, show what is sometimes described as a "paradoxical" reaction to benzodiazepines, acting with more unrest instead of sedation. An alcohol abuse history is a documented risk factor for this occurrence, and such patients sometimes respond to flumazenil [27]. Therefore, we do favor the use of flumazenil in cases in which benzodiazepine delirium is suspected, regardless of the phenotypic subtype of the syndrome, as long as a correlative history and physical examination are consistent with the differential diagnostic possibility.

Specific to benzodiazepine activity in the treatment of AWS, only one of our patients who received flumazenil after abstinence day 4 experienced adverse events. In light of the typical course of alcohol withdrawal whose severity peaks before day 5 [28], this finding has changed our practice. We now administer diagnostic flumazenil to most cognitively impaired AWS patients on abstinence day 5, and if their mental status improves, we discontinue sedatives and repeat flumazenil every hour as needed, which may shorten hospital length of stay - an outcome worthy of further study in prospective fashion.

Our favorable side effect profile appears better than those reported in previous studies and may be secondary to the exposure of some patients to other sedatives which stimulate the GABA-A receptor. Additionally, our initial flumazenil doses $(0.5 \mathrm{mg}$ or less) may incompletely antagonize the pool of GABA-A receptors in the adult CNS. Previous non-human pharmacokinetic studies have found adverse events may be more prevalent with higher doses of flumazenil administration [29]. But occasionally, a patient receiving $0.5 \mathrm{mg}$ of flumazenil may require more frequent dosing than hourly. For such a patient, our practice is to increase the administered flumazenil up to $1 \mathrm{mg}$ (in most instances with good effect), although a prior dose duration of effect study after continuously infused midazolam suggests the effects of flumazenil are prolonged more meaningfully at 3-mg doses [20]. However, the rare instances of seizures precipitated by flumazenil in bedside clinical practice appear to correlate with doses greater than $1 \mathrm{mg}$ at a time [19].

In addition to attention to appropriate dosing to avoid seizures, our clinical experience suggests patients with AWS who have seizures do so on abstinence days 1-2. Most of our patients received flumazenil later in the course of AWS, which may lessen their risk for seizure. However, we still used flumazenil earlier in the course of AWS when benzo delirium was suspected without serious adverse effects.

The primary purpose of this study was to demonstrate our experience using flumazenil to diagnose and treat benzodiazepine delirium during the course of AWS; guided by bedside assessment, intermittently dosed flumazenil was shown to have a favorable risk-benefit ratio. We do not employ flumazenil infusions, because we suspect they prolong treatment length and have not seen uniform administration patterns. Future studies may find flumazenil use can shorten length of hospital stay as well as decrease complications, including, but not limited to, injuries to patients and hospital staff.

\section{Limitations}

Limitations to our study include the retrospective study design $[30,31]$, non-blinded chart reviewers, and some missing and/ or potentially missing data. The retrospective study design was strengthened by one reviewer training the other reviewers then sampling/confirming data abstraction periodically after competency established. We also strengthened the retrospective study design by utilizing a data collection tool.

Limited data was available about amount and duration of ethanol abuse and history of alcohol withdrawal. If a patient was without detectable ethanol and/or serum ethanol was not collected, abstinence day was based on patient's report, supported by number of days in hospital. Limited data was available about pre- and post-flumazenil Riker scores, but often these patients had clearly documented response but not in the Riker format. We had hoped to document preand post-flumazenil vital signs, but found during data collection they were not consistently collected at both times with the second at the time of flumazenil peak effect.

We did not have patients on continuous EEG monitoring during the time of flumazenil administration, but were diligent when reviewing physician and nursing notes surrounding flumazenil administration for documentation of seizures or other adverse events. All patients were monitored on telemetry, and many had 1:1 behavior monitors; these practice parameters increase the likelihood a seizure or arrhythmia would be captured.

\section{Conclusions}

On the basis of our experience, noting both a demand for bedside expertise and the lack of some objective data in this retrospective study, flumazenil administration may effectively diagnose and treat benzodiazepine delirium occurring during the course of AWS treatment without serious or lifethreatening adverse events. Patients with a purely hyperactive delirium may be less likely to have a positive response to flumazenil than other delirium subtypes, but a majority of agitated patients with benzodiazepine delirium may show neurobehavioral improvement. During the treatment of AWS, if delirium is present on day 5 of abstinence without autonomic or peripheral nervous hyperreactivity, consider 
administering a test dose of flumazenil to diagnose and potentially treat benzodiazepine delirium.

Acknowledgments The authors thank Helen Houpt MSLS, AHIP, for assistance with editing and manuscript preparation, Elizabeth Morgan for general library services and assistance obtaining specified references, and Betty Ruppert and Amanda Cresswell RN, MSN, CMSRN, for assistance with study coordination and chart review.

Conflict Delineations The authors report no conflict delineations.

Sources of Funding none

\section{References}

1. Short TG, Young KK, Tan P, Tam YH, Gin T, Oh TE (1994) Midazolam and flumazenil pharmacokinetics and pharmacodynamics following simultaneous administration to human volunteers. Acta Anaesthesiol Scand 38:350-356

2. Ma JD, Lawendy NM, Fullerton T, Snyder PJ, Nafziger AN, Bertino JS Jr (2009) Effect of intravenous flumazenil on oral midazolam pharmacokinetics and pharmacodynamics for use as a cytochrome P450 3A probe. Int J Clin Pharmacol Ther 47:111-119

3. Jones RD, Chan K, Roulson CJ, Brown AG, Smith ID, Mya GH (1993) Pharmacokinetics of flumazenil and midazolam. Br J Anaesth 70:286-292

4. Oliver FM, Sweatman TW, Unkel JH, Kahn MA, Randolph MM, Arheart KL et al (2000) Comparative pharmacokinetics of submucosal vs. intravenous flumazenil (Romazicon) in an animal model. Pediatr Dent 22:489-493

5. Mandema JW, Gubbens-Stibbe JM, Danhof M (1991) Stability and pharmacokinetics of flumazenil in the rat. Psychopharmacology (Berlin) 103:384-387

6. Kretz FJ, Loscher W, Peisdersky B, Kraft A, Eyrich K (1990) Flumazenil (Anexate): pharmacodynamics, pharmacokinetics, indications and contraindications. Med Klin (Munich) 85:156-162, 69

7. Klotz U, Kanto J (1988) Pharmacokinetics and clinical use of flumazenil (Ro 15-1788). Clin Pharmacokinet 14:1-12

8. Klotz U (1988) Drug interactions and clinical pharmacokinetics of flumazenil. Eur J Anaesthesiol Suppl 2:103-108

9. Kim YJ, Lee H, Kim CH, Lee GY, Baik HJ, Han JI (2012) Effect of flumazenil on recovery from anesthesia and the bispectral index after sevoflurane/fentanyl general anesthesia in unpremedicated patients. Korean J Anesthesiol 62:19-23

10. Karakosta A, Andreotti B, Chapsa C, Pouliou A, Anastasiou E (2010) Flumazenil expedites recovery from sevoflurane/remifentanil anaesthesia when administered to healthy unpremedicated patients. Eur J Anaesthesiol 27:955-959

11. Henthorn KM, Dickinson C (2010) The use of flumazenil after midazolam-induced conscious sedation. Br Dent J 209:E18

12. Potokar J, Coupland N, Glue P, Groves S, Malizia A, Bailey J et al (1997) Flumazenil in alcohol withdrawal: a double-blind placebocontrolled study. Alcohol Alcohol 32:605-611

13. Veiraiah A, Dyas J, Cooper G, Routledge PA, Thompson JP (2012) Flumazenil use in benzodiazepine overdose in the UK: a retrospective survey of NPIS data. Emerg Med J 29:565-569
14. Kreshak AA, Cantrell FL, Clark RF, Tomaszewski CA (2012) A poison center's ten-year experience with flumazenil administration to acutely poisoned adults. J Emerg Med

15. The Flumazenil in Benzodiazepine Intoxication Multicenter Study Group (1992) Treatment of benzodiazepine overdose with flumazenil. Clin Ther 14:978-995

16. Weinbroum A, Rudick V, Sorkine P, Nevo Y, Halpern P, Geller E et al (1996) Use of flumazenil in the treatment of drug overdose: a double-blind and open clinical study in 110 patients. Crit Care Med 24:199-206

17. Soleimanpour H, Ziapour B, Negargar S, Taghizadieh A, Shadvar K (2010) Ventricular tachycardia due to flumazenil administration. Pak J Biol Sci PJBS 13:1161-1163

18. Rasimas JJ, Smolcic EE, Cressell A, Sachdeva K, Donovan JW (2010) Bedside toxicologic experience with physostigmine and flumazenil. Clin Toxicol Abstract

19. Ngo AS, Anthony CR, Samuel M, Wong E, Ponampalam R (2007) Should a benzodiazepine antagonist be used in unconscious patients presenting to the emergency department? Resuscitation 74:27-37

20. Dunton AW, Schwam E, Pitman V, Leese P, Siegel J (1988) The relationship between dose and duration of action of intravenous flumazenil in reversing sedation induced by a continuous infusion of midazolam. Eur J Anaesthesiol Suppl 2:97-102

21. Camus V, Gonthier R, Dubos G, Schwed P, Simeone I (2000) Etiologic and outcome profiles in hypoactive and hyperactive subtypes of delirium. J Geriatr Psychiatry Neurol 13:38-42

22. Camus V, Burtin B, Simeone I, Schwed P, Gonthier R, Dubos G (2000) Factor analysis supports the evidence of existing hyperactive and hypoactive subtypes of delirium. Int J Geriatr Psychiatry 15: 313-316

23. Stagno D, Gibson C, Breitbart W (2004) The delirium subtypes: a review of prevalence, phenomenology, pathophysiology, and treatment response. Palliat Support Care 2:171-179

24. Khan BA, Guzman O, Campbell NL, Walroth T, Tricker J, Hui SL et al (2012) Comparison and agreement between the Richmond agitation-sedation scale and the Riker sedation-agitation scale in evaluating patients' eligibility for delirium assessment in the ICU. Chest 142:48-54

25. Riker RR, Picard JT, Fraser GL (1999) Prospective evaluation of the sedation-agitation scale for adult critically ill patients. Crit Care Med 27:1325-1329

26. Riker RR, Fraser GL (2001) Monitoring sedation, agitation, analgesia, neuromuscular blockade, and delirium in adult ICU patients. Sem Respir Crit Care Med 22:189-198

27. Mancuso CE, Tanzi MG, Gabay M (2004) Paradoxical reactions to benzodiazepines: literature review and treatment options. Pharmacotherapy 24:1177-1185

28. Maldonado JR (2010) An approach to the patient with substance use and abuse. Med Clin N Am 94:1169-1205, x-i

29. Lheureux P, Vranckx M, Leduc D, Askenasi R (1992) Flumazenil in mixed benzodiazepine/tricyclic antidepressant overdose: a placebo-controlled study in the dog. Am J Emerg Med 10:184-188

30. Gilbert EH, Lowenstein SR, Koziol-McLain J, Barta DC, Steiner J (1996) Chart reviews in emergency medicine research: where are the methods? Ann Emerg Med 27:305-308

31. Worster A, Bledsoe RD, Cleve P, Fernandes CM, Upadhye S, Eva K (2005) Reassessing the methods of medical record review studies in emergency medicine research. Ann Emerg Med 45:448-451 